\title{
Nuclear Cysteine Cathepsins in Thyroid Carcinoma
}

Alaa al-Hashimi' ${ }^{1}$, Sofia Tedelind ${ }^{1}$, Joanna Szumska1, N. Kim Dierkes ${ }^{1}$, Alexandra Pinzaru', Roberta Burden ${ }^{2}$, Christopher Scott ${ }^{2}$, Dagmar Führer ${ }^{3}$, and Klaudia Brix ${ }^{1}$

\section{Introduction}

Cysteine cathepsins play crucial roles in thyroid physiology through thyroglobulin processing for liberation of thyroid hormones by extra- and intracellular means. However, in thyroid cancer, cathepsin B is over-expressed and secreted into the extracellular space, thus promoting migratory phenotypes of thyroid carcinoma cells through excessive extracellular matrix degradation [1]. In addition, we have shown that $\mathrm{N}$-terminally truncated forms of cathepsin $\mathrm{V}$, which lack the signal peptide and parts of the pro-peptide, are localized to the nuclei of anaplastic thyroid carcinoma cells, while cathepsin $L$ is present within endo-lysosomes as expected [1]

Here, localization patterns of cathepsins $B$ and $V$ were examined in a variety of human thyroid carcinoma cell lines in comparison to normal thyroid epithelial cells by immunofluorescence studies.
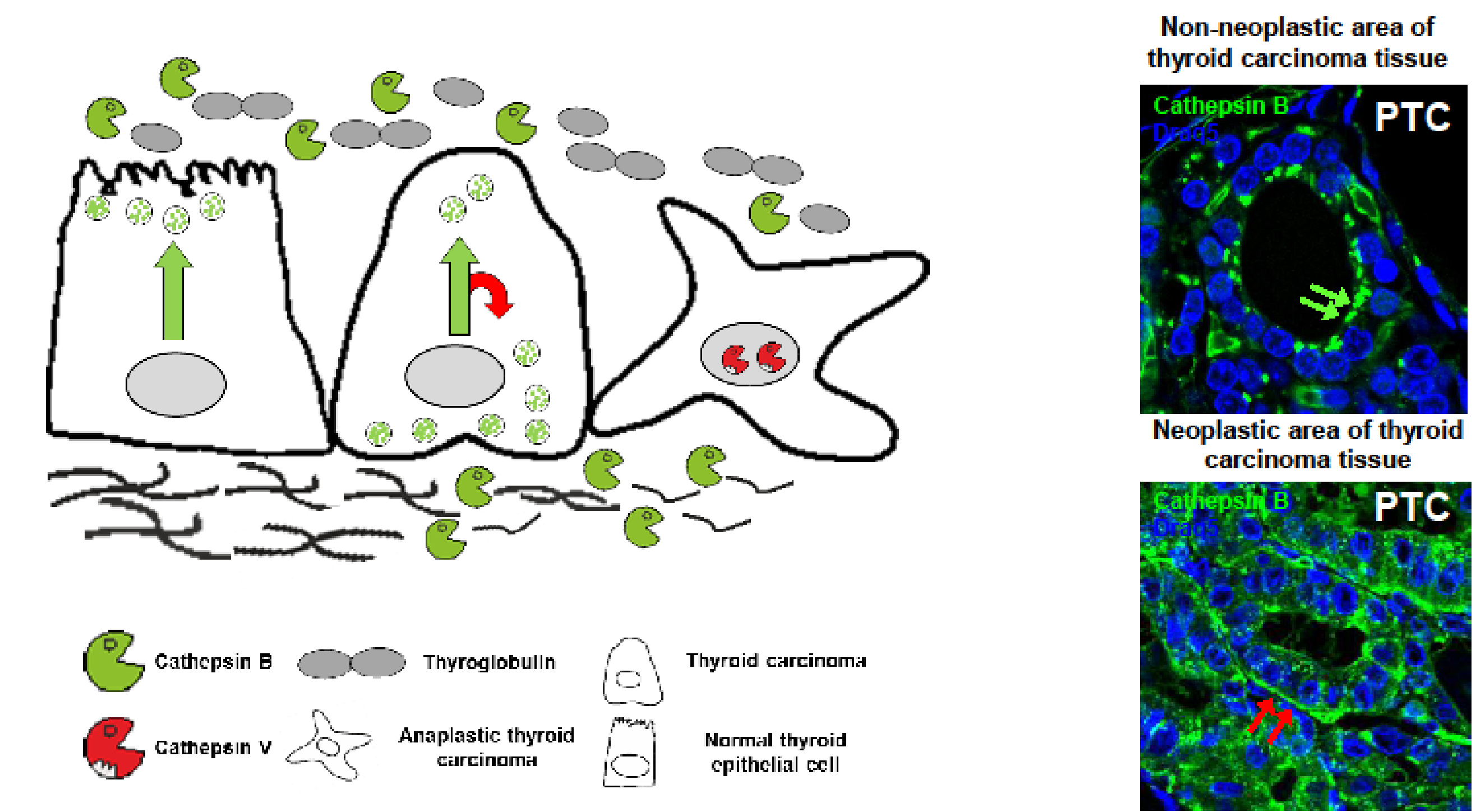

\section{Characterization of Normal Thyroid Epithelial and Carcinoma Cell lines}

Nthy-ORI 3-1 and HTh74 cells labelled with FITC-phalloidin revealed stress fibers and lamellipodia, suggesting the ability of these cells to adhere to the substratum and to migrate, respectively. KTC-1 cells exhibited cortical Factin, actin fibers, and a fine actin network. FTC-133 cells showed F-actin structures in the cytoplasm and prominent cortical F-actin, indicating that these cells lost their ability to tightly adhere to the extracellular matrix.

Nthy-ORI-3-1, KTC-1, FTC-133 and HTh74 cell lines represent different stages during epithelial-to-mesenchymal transition. KTC-1 cells displayed almost typical epithelial sheets composed of polarized cells. FTC-133 cells lacked cell-cell contacts and featured high levels of the mesenchymal marker vimentin. Mesenchymal characteristics including loss of intercellular adhesion and expression of vimentin was also observed in Nthy-ORI-3-1 and HTh74 cells. Nuclear-DNA was counter-stained with Draq5. Scale bars
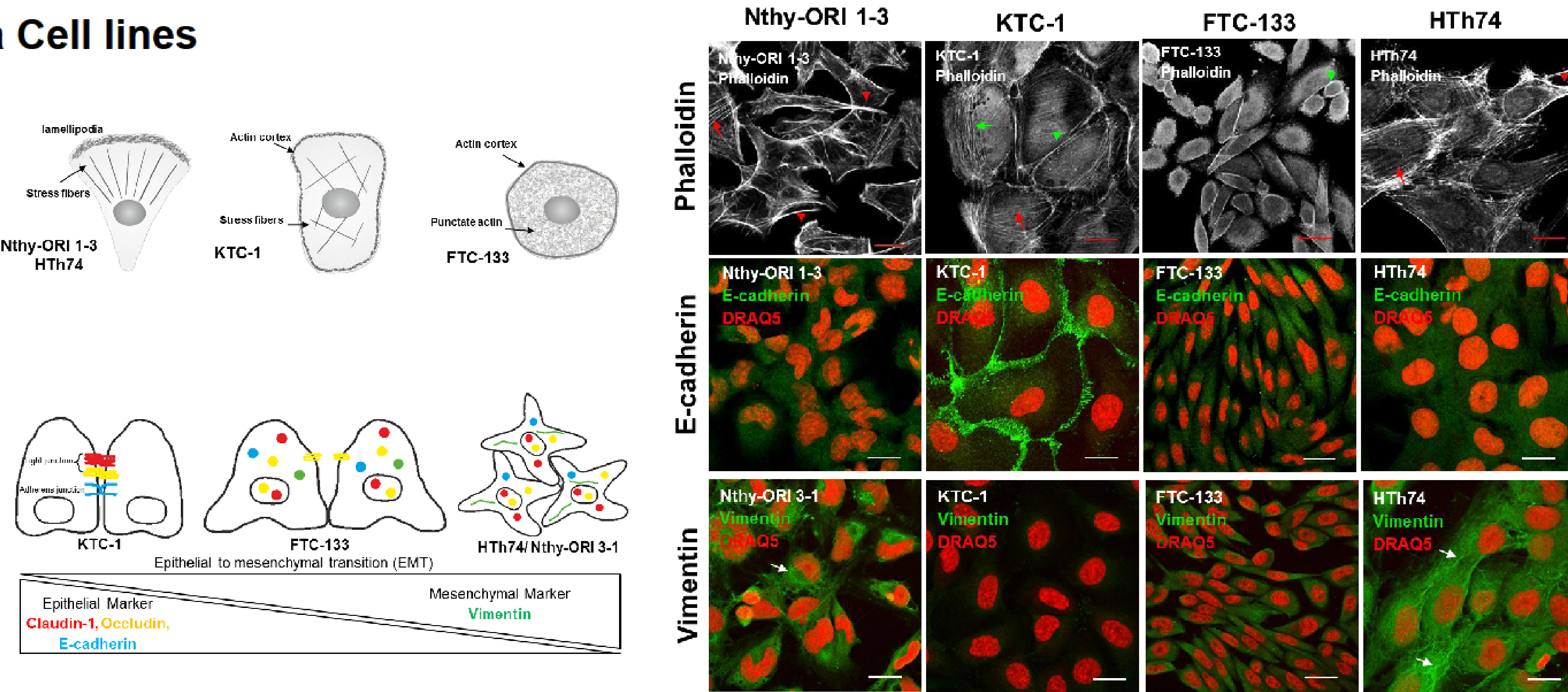
represent $20 \mu \mathrm{m}$.
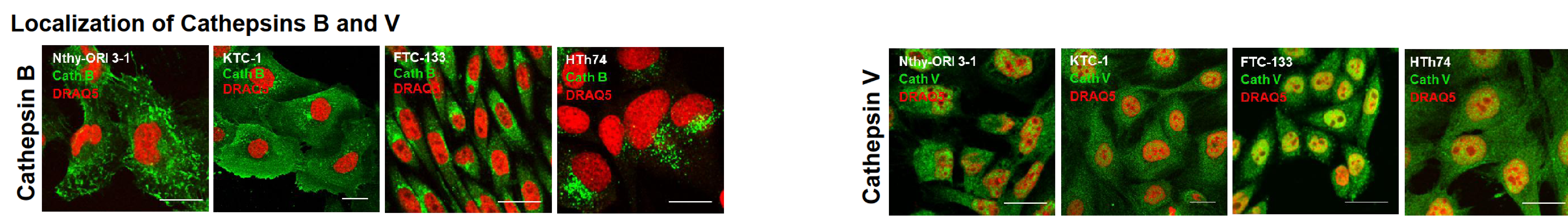

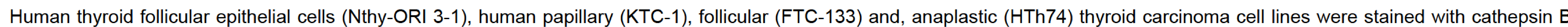

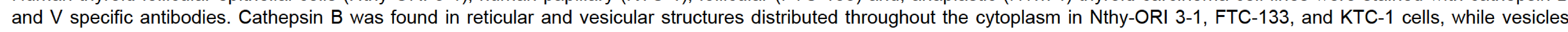

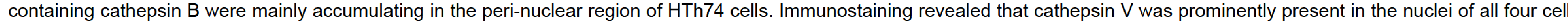
lines as well as in vesicles distributed throughout the cytoplasm. Nuclear-DNA was counter-stained with Draq5. Scale bars represent $20 \mu \mathrm{m}$.

Cathepsins B and V Localization During Cell Cycle of KTC-1 Cells
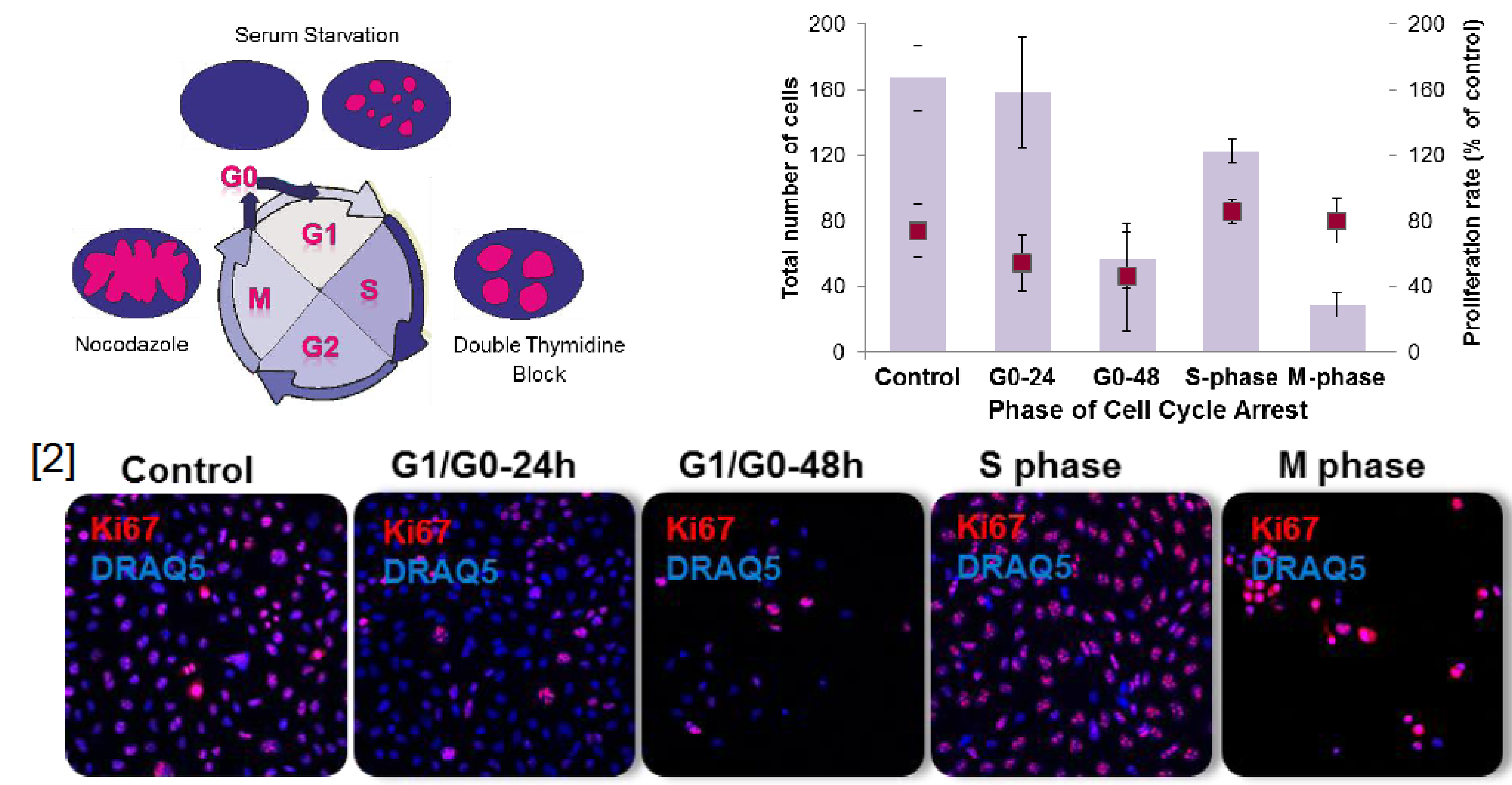

Arrest of KTC-1 cells in G1/G0 phase was by incubation in serum-free medium for 24 and $48 \mathrm{~h}$ For synchronization in early $S$ phase, cells were incubated in thymidine for 18 and $17 \mathrm{~h}$ interrupted by a 9-h-incubation in complete medium. For G2/M arrest, cells were treated with thymidine for 18 $\mathrm{h}$, and released for $3 \mathrm{~h}$ by incubation in complete culture medium, before they were incubated with nocodazole for another $12 \mathrm{~h}$. Cells were immunolabeled with Ki67 as proliferation marker, cathepsin B and V specific antibodies, and Draq5 counter-staining nuclear DNA.
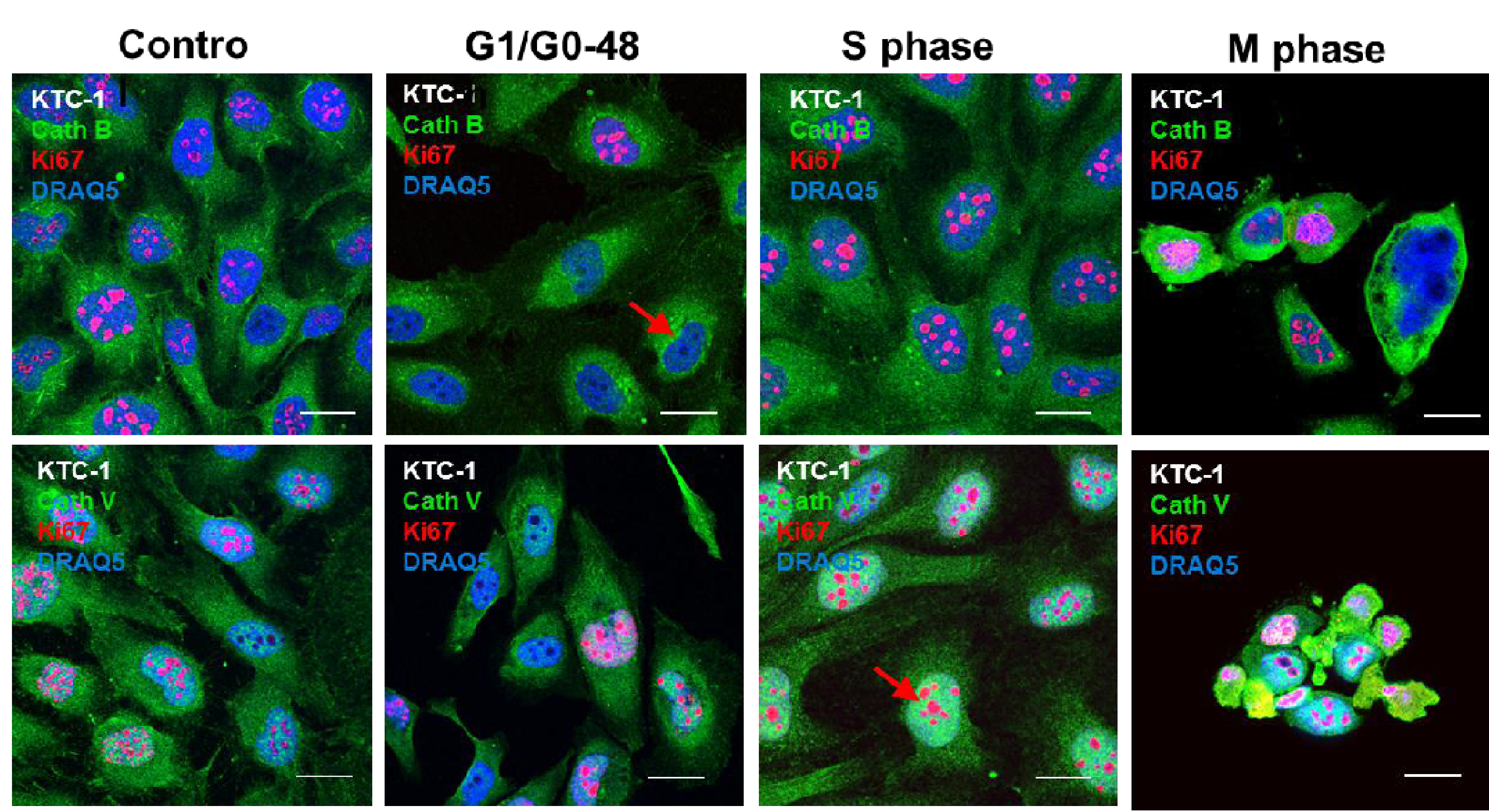

Immunolabeling of synchronized KTC-1 cells revealed cathepsin B staining in ERlike reticular structures as well as in the Golgi apparatus and in endo-lysosomal vesicles, but no prominent nuclear staining was observed. In contrast, cathepsin V was also present in the nuclei throughout the cell cycle. In particular, proliferative cells featured nuclear cathepsin $\mathrm{V}$ signals. The strongest nuclear staining of cathepsin $\mathrm{V}$ was detected in the nuclei of KTC-1 cells in S phase.

\section{Conclusions}

$>$ Human thyroid epithelial cells in vitro (Nthy-ORI 3-1) are less polarized than in situ.

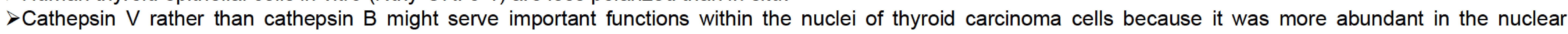
compartment than cathepsin B.

$>$ We hypothesize that cathepsin $\mathrm{V}$ is involved in de-regulation of cell cycle progression during thyroid tumorigenesis 